\title{
Leaf senescence and its regulation with phytohormones and essential elements: An overview
}

\author{
Shatrupa Singh ${ }^{1}$, Madhulika Singh ${ }^{1,2}$, Sanskriti Bishtt ${ }^{1}$, Jai Gopal Sharma ${ }^{1 *}$ \\ ${ }^{1}$ Department of Biotechnology, Delhi Technological University, Delhi, India. \\ ${ }^{2}$ Department of Botany, SSN College, University of Delhi, Delhi, India.
}

\begin{tabular}{l}
\hline ARTICLE INFO \\
\hline Article history: \\
Received on: June 16, 2021 \\
Accepted on: October 28, 2021 \\
Available Online: February 15, 2022 \\
\hline
\end{tabular}

Key words:

Aging, cell death, oxidative stress, remobilization of nutrient

\begin{abstract}
Leaf senescence is a crucial developing phase that requires the orderly disassembly of macromolecules in order to transport the nutrients from leaves into other organs and is life-threatening for plants capability. The leaf senescence is the result of a multifaceted and highly regulated mechanism involving the corresponding activities of several pathways. A lot of progress has been made recently in understanding signaling pathways of senescence, as well as how to complete the orderly process of degeneration. This paper mainly covers recent developments in the senescence of leaf and describes the function of phytohormones and essential elements from the molecular network dynamics.
\end{abstract}

\section{INTRODUCTION}

The term "aging" can be defined as the increase in age, leading to age-dependent degradation of tissues and species in later stages of life in both plants and animals [1]. However, senescence refers to the highly regulated expression of gene and the presence of cellular degradation in plants and animals with somatic/germline. Two forms of aging exhibit in plants: mitotic and post mitotic senescence [1,2]. Mitotic senescence occurs in meristematic tissue when cell or replication division is stopped. Post-mitotic ensues in plant organs such as leaves and floral petals after the cell differentiation and development which follows a dynamic and programmed deteriorating progression [3]. This is crucial and normally occurs in various plant tissue forms, viz., leaves, reproductive organs (stamens and style), root cap, and germinating seeds. Senescence and programmed cell death (PCD) both reflect the mechanisms that contribute to loss of individual cells. The senescence can therefore also be considered as one of the examples of PCD [4].

*Corresponding Author

Jai Gopal Sharma, Department of Biotechnology, Delhi Technological

University, Delhi, India.E-mail: sharmajaigopal @dce.ac.in
The process of leaf senescence develops regularly, in which the plant structures and biomolecules are broken down and then transported to various other plant parts like fruits, seed, and apical leaves. Leaf senescence is based on age-dependent and accelerates in transition from vegetative to reproductive development.

Senescence process is genetically regulated which involves significant changes in the pattern of expression of genes leading to cell degradation and reallocation of resulting products to the newly developed organs. Several senescence-associated genes, encode enzymes that participate in protein degradation and emphasize the importance of nitrogen recycling. In several species, mutants that show delayed leaf senescence have been identified and are very useful in the study of cell maturation and cell death. All these mutants maintain the color of leaves for a prolonged period of time. Functional mutants which are combined with a delay in senescence and metabolic ability preservation are conveniently distinguished from those that maintain green color but have the behavior of natural aging. The progression and initiation of leaf senescence process is correlated with environmental factors as well as developmental cues (Fig. 1). In fact, senescence may be caused by darkness, abiotic stresses, and microorganisms in otherwise young leaves [5]. 
The plant responds to these inputs are mediated by endogenous molecules for signaling pathway such as phytohormones, reactive oxygen species (ROS) and other signals dependent on redox, leading to comprehensive genomic, physiological, and metabolic reprogramming. The objective of this review is to critically discuss the molecular processes that incorporate different environmental signals through pathways of senescence and address the role of some essential nutrients (P, S, Fe, and $\mathrm{Ni}$ ) in leaf senescence.

\section{HISTORY}

Since 1961, plant senescence is mystery of biology, where Carl Leopold sensed the main factors that could govern them. The discovery of several hormones allowed a better understanding of phenomenon, in particular how hormones controlled the growth, as in leaf. After the discovery of the molecular bases of genetics, several specialists attempted to connect the hormone activity with the molecular genetics work but only during the 1990s, after the discovery of specific senescence-associated genes (SAGs), the role of promoting-senescence hormones and retardingsenescence hormones was elucidated, with the help of the plant species Arabidopsis thaliana and its mutants. The history of leaf senescence in chlorophyll degradation had its paradigmatic model, a mechanism that was a mystery until 1991, where degradative products started to be isolated and chemically defined [6].

Various hypotheses have been developed that senescence was a result of the membrane integration breakdown. Eilam found that bean leaves senescence was linked to potassium leakage from leaves and associated growth of the apparent free space. Poovaiah and Leopold [7] demonstrated a hydraulic permeability increased and apparent free space changes in corn leaf discs during senescence.

\section{MODIFICATIONS IN THE CHLOROPLAST DURING THE LEAF SENESCENCE}

Leaf reflects the autotrophic plants and is the only source of food since they use light to fix carbon. They are photosynthetically

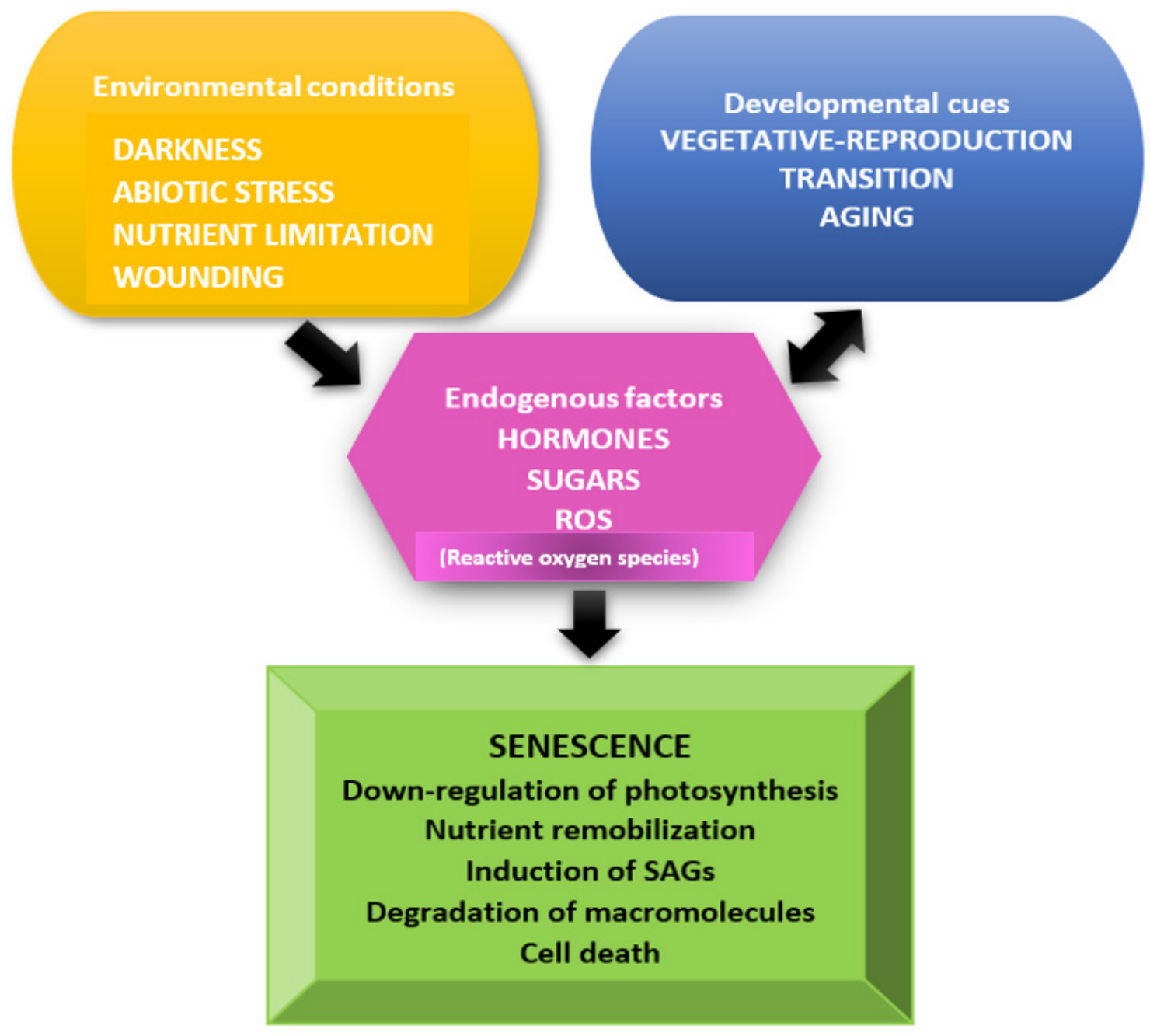

Figure 1: Summary of leaf senescence. In last stage of leaf development, exogenous and endogenous signals bring the plant's life cycle to an end. Biotic, abiotic, and nutritional conditions influence senescence, while endogenous factors (hormones) represent the most significant factors of controlling senescence [5]. 
competent and accumulate nutrients during the period of their growth and development. Then leaves reach the stage of senescence, following their death. Leaf senescence is a closely controlled biological process that includes the process of "wear and tear" during aging [8]. Leaf cells undergo drastic metabolic and biochemical changes during senescence, including a shift to catabolism, resulting in the transfer of nutrients to newly developing organs. In order to turn carbon assimilation into nutrient remobilization, cellular structure such as chloroplasts are degraded. In chloroplast, chlorophyll is highly degraded, resulting in yellowing of leaf that is the most noticeable sign of plant senescence. Furthermore, Arabidopsis, pheophytinase $(\mathrm{PPH})$ is significant enzyme that is associated with degradation of chlorophyll. Residues of the amino acid forming the catalytic PPH triad are also determined in combination with site-directed mutagenesis through the silicon modeling of the 3D structure [9].

\section{LEAF SENESCENCE CONTROL MECHANISM}

Over the last couple of decades, the area of research on leaf senescence has expanded considerably, but the methods used are still different for physiological and molecular investigations. To conduct senescence analyses in Arabidopsis [10] formal guidelines have been established for growing and differentiating leaves, one of which is an experimental design for using plant physiological, biochemical, and molecular biomarkers.

The onset of senescence in the leaves is caused by internal or external causes, such as plant age, or exposure to prolonged darkness, high temperature, or other biotic stressors. During leaf senescence, highly sequential changes occur, with emphasis on structure, gene expression, and metabolism, which are now known as three stages: initiation, reorganization, and termination [11]. However, the mechanisms of initiation can vary under different conditions. Processes of degradation and disassembly occur during degeneration while cell death characterizes termination phase [12].

\subsection{Initiation Stage}

The phase of initiation is early signals which leads to gene expression changes and process of senescence. Leaf senescence are caused by environmental stresses in some species and not in others, and rely on the size, magnitude of the stresses, and the growth stage in which they occur. Therefore, it can be regarded as another mechanism by which some plants have developed under pressure and must be examined in relation to other stress reactions which also offer plant defense. Although certain characteristics of drought stress and the signal translation pathways contributing to expression of gene have been characterized. The modifications in the expressions of gene have resulted in alterations in plant regulators endogenous concentrations, such as cytokinin (CTK), abscisic acid (ABA), ROS which control the expression of senescence-linked genes [13] (Fig. 2).

\subsection{Reorganization Stage}

Significant changes in the cell structure and the metabolic process during leaf senescence occurs throughout the reorganization process (also named as trans differentiation or degeneration), including deterioration of chlorophyll, decline in photosynthetic activity and cell integrity disassembly. All these alterations are associated with the remobilization of nutrient. The source-to-sink evolution thus plays an important part through the reorganization process, and major changes occur in the senescing leaves. This phase's events tend to be degenerative in nature. Nevertheless, it is now recognized that they arise from a reorganization phenomenon (or trans differentiation), instead of a degenerative mechanism, resulting from a closely regulating of particular genes [14].

Reorganization process is hence an active process and is controlled by a complex signal-regulatory mechanism that gets turned on during the initialization phase. Cellular CTK, ABA, salicylates, and ROS can govern the leaf senescence process. Certain processes, including decrease in photosynthesis and chlorophyll degradation, oxidative stress, and cellular deterioration, are triggered concurrently while others, including lipid peroxidation and loss of integrity, ensue when hormonal and ROS concentrations exceed a particular level [13].

\subsection{Terminal Phase of Leaf Senescence}

In the last phase, the apoptosis marks such as condensation of chromatin and DNA laddering are observed, as the leaves have almost fully turned yellow. This means that the senescence of leaf involves processes of apoptosis-like cell death. The hypersensitive response (HR) is another cell-death mechanism in higher plants that is involved in pathogen defense. The leaf senescence and defensive responses, which involve HR, have some similarities in molecular and physiological terms. For example, the activation of genes associated with pathogenesis and the accumulation of salicylic acid (SA) and ROS include both procedures. Therefore, leaf senescence and cell mortality as a part of HR, can at least share a similar signaling pathway. Accelerated death due to mutations are identified to occur in leaves of plants that are particularly old, like one that can cause the loss of defensive ability. Some of these mutants have flaws in the senescence program [12].

\section{MAJOR PLAYERS IN MODULATION OF SENESCENCE}

Phytohormones such as ethylene (ET), jasmonic acid (JA), SA, and ABA, as well as to a lesser amount brassinosteroids, are main players in the regulation of senescence. CTKs, auxins, and gibberellins may be thought of as senescence-delaying hormones. Transcription factors are essential components of all signaling networks because they direct the expression of receptive genes. They also serve as nodal regulators of developmental or defense reactions. ROS control senescence and cell death, as well as the early conditions after a pathogen is recognized. Because to the complexity as well as cross-linking of the signaling networks underlying these key players, only those modules important for senescence, as well as nodes of interaction and integration, will be highlighted.

\subsection{Phytohormones}

\subsubsection{Ethylene}

ET is a gaseous plant hormone which play a vital role in plant growth development mechanisms and reactions to environmental 
stimuli. It exerts opposing effects in its contact with necrotrophic and hemibiotrophic pathogen ET is mainly involved in senescence and PCD during HR. Cell death and aging are favorable to necrotrophic pathogens. As a virulence mechanism, many necrotrophic or hemibiotrophic pathogens induce or synthesize ET as a consequence [15].

ET has a crucial function in leaf senescence control. ET activates senescence, especially in sensitive species. During the first stage of leaf development, ET production is high, then falls until the leaf achieves maturity, when it is fully extended, before increasing again during the early stages of senescence onset. At the molecular level, it's been discovered that various genes in the same family code for ET biosynthesis enzymes that are active throughout leaf growth, and that their expression is timed. Biosynthesis can take place anywhere on the plant, at any stage of leaf growth [16]. Aside from its physiological activities in various developmental phases, ET was once thought to be a stress hormone since it is produced in response to a number of stress signals, including mechanical wounds, chemicals and metals, drought, high temperatures, and pathogen infection [17,18]. Stress-induced ET production is usually regulated by speeding up the conversion of S-Adenosyl methionine to 1-aminoacyclopropane 1-carboxylate synthase (ACC), indicating that ACC synthase expression is the main target of regulation. Stimulation for ET production has been found to include the formation of ROS in response to environmental stressors such as ozone, UV irradiation, and wounding [19-21].
Although for the ripening of several fruits' ET is required, it has also been linked to the senescence of certain plant leaves. Plants which are subjected to ET experience premature senescence, with aged leaves turning yellow. The initiation of senescence in the leaves of an Arabidopsis ET-insensitive mutant (Etr1) appeared delayed. However, the leaves of these mutants ultimately senesce, showing that ET's function in leaf senescence is not necessary, but rather affects the time of senescence in Arabidopsis. To corroborate this concept, when ET biosynthesis in tobacco and tomato plants was genetically suppressed, the plants showed a delay in the start of leaf senescence. Similarly, there was delayed leaf senescence in an antisense tomato plant which had low amounts of ET. Furthermore, several mutant lines of Arabidopsis that have been described to delay senescence, display defects in the gene pathways for ET signaling. Nevertheless, senescence happens naturally after the process starts. To react to the ET signal, leaves must be a certain age; ET-treated young leaves should not senesce [14].

\subsubsection{Jasmonic acid}

Similar to ET, senescence, cell death, and tolerance to necrotrophic pathogens are all controlled by JA. During lipid peroxidation, JA is produced in chloroplasts and peroxisomes. The physiologically active compound, jasmonoyl-isoleucine, works by depressing the expression of its target genes [14].

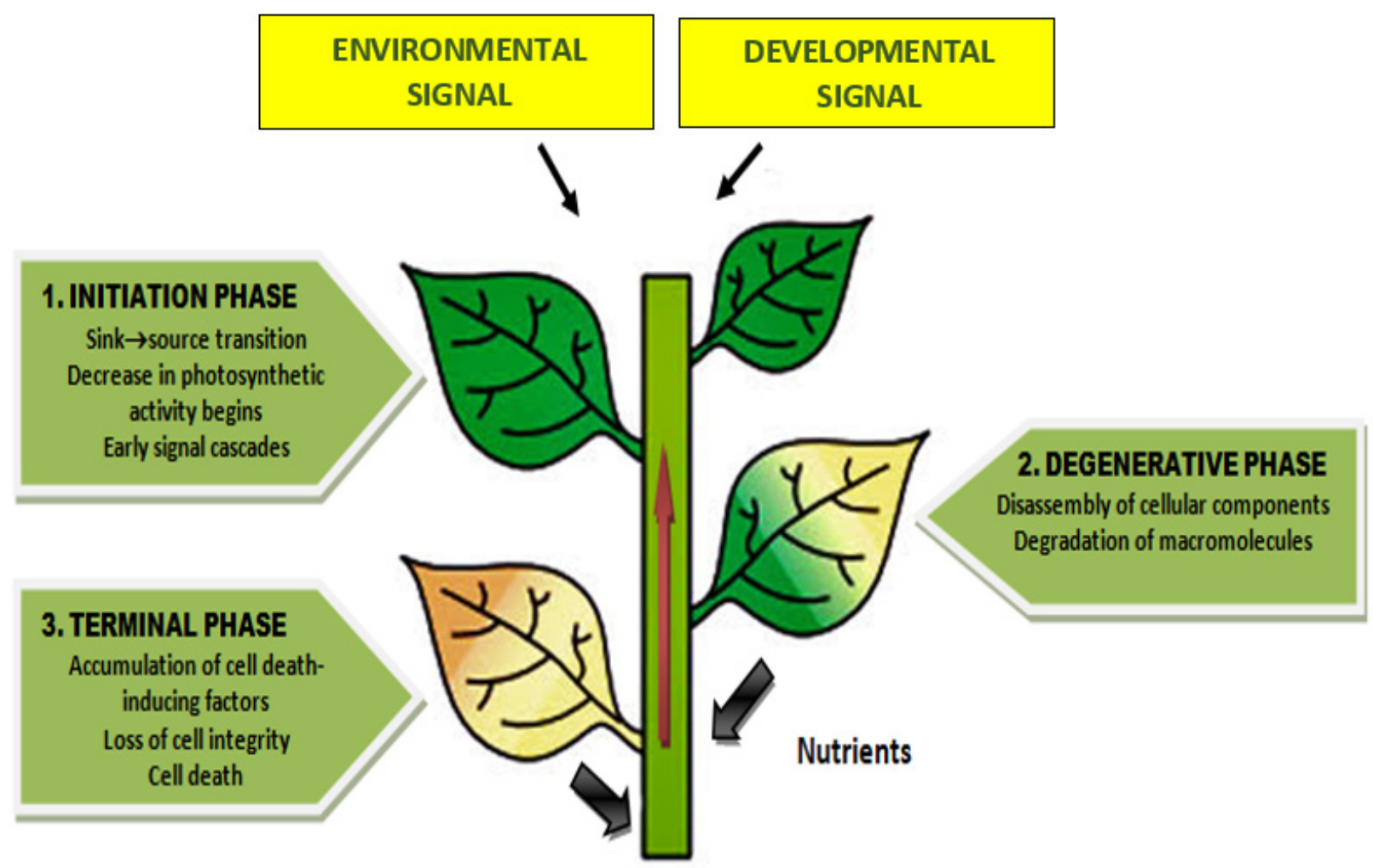

Figure 2: An outlined route to leaf senescence [12]. 
JA and associated compounds have been engaged in a number of plant responses, including wounding and pathogen infection. Additionally, JA has been related to senescence. It was demonstrated that exogenous JA or methyl jasmonate (MeJA) treatment of barley leaves resulted in chlorophyll loss suggesting that JA enhance senescence process. Additionally, it was demonstrated that a few enzymes engaged in biosynthesis of JA showed increased expression during senescence, and JA levels increased. MeJA therapy increases the expression of a potential chlorophyllase gene, according to studies on genes involved in the chlorophyll degradation pathway (AtCHL1) [15].

\subsubsection{Salicylic acid}

SA is a protective hormone. It plays an important role during plant development in non expressor of PR-genes (NPR1) and phytoalexin-deficient cells, SA induces developmental senescence gene (pad4). It has also been shown to activate the transcription factor WRKY53, which is a key positive regulator of ageing and pathogen defense. The backbone of SA-mediated defense responses is the signaling mechanism that leads to the activation of pathogenesis-related (PR) genes and systemic acquired resistance (SAR) effector-triggered immunity [22]. HR encapsulates biotrophic pathogens at the outbreak site, preventing them from spreading to healthier areas of the plant.

\subsubsection{Abscisic acid}

In the control of senescence and the regulation of pathogen defense, ABA can play a wide range of roles. Although ABA is thought to encourage senescence, its function in pathogen protection for both biotrophic and necrotrophic pathogens remain unknown. Crosstalk exists with a variety of other signaling mechanisms and cellular processes, including JA, ET, SA, CTK and sugar signaling, ROS production and signaling, and autophagy [23-25].

\subsubsection{Auxins}

Despite the fact that all of the above phytohormones are believed to stimulate ageing, auxins, CTK and gibberellins are thought to be senescence-delaying hormones [26]. Specifically, indole acetic acid, control plant growth and production in a variety of ways. Auxins are generated in young tissues by two distinct pathways from tryptophan, emphasizing their importance. Additionally, auxin efflux carriers with a polar distribution in cells aid polar auxin transport. The existence of fine auxin gradients that structure plant architecture is critical [22]. Auxins perform a related role by regulating the expression of several auxin-responsive transcription factors that regulate a variety of leaf senescence processes. Furthermore, increased expression of YUC6, a flavin-containing monooxygenase which catalyzes the rate-limiting stage in auxin biosynthesis, was found to be beneficial in delaying senescence in transgenic Arabidopsis plants [5].

\subsubsection{Cytokinin}

CTK have the ability to delay senescence in plants and detached leaves by avoiding chlorophyll loss and metabolic activity disruption. On the other hand, a decrease in the CTK pool is sometimes associated with decline in photosynthetic response and a rise in senescence [27]. One of the most thoroughly researched hormones associated with the temporally distinct stages of development is CTK, a hormone that stimulates cell growth and division during leaf growth processes and it slows the progression of leaf senescence via cell proliferation. The CTK receptors Arabidopsis histidine kinase 2, 3, and 4 are involved in leaf growth and senescence. Additionally, CTK response factors are downstream components of CTK receptors that regulate leaf growth and senescence by mediating CTK signaling [28].

CTK and ET induce comparable responses in early seedling development despite having opposing functions in senescence regulation. The ET-mediated triple response is seen in dark-grown Arabidopsis seedlings administered with small doses of CTKs. The lack of a three-fold response in the presence of CTK led to the isolation of CTK -insensitive mutants (cin). The CIN5 gene, which encodes 1-amino-cyclopropane-1-carboxylate synthase, belongs to the Arabidopsis gene family. The persistent increase in ET production reported in response to low levels of CTK is mostly due to this first enzyme in the ET biosynthesis pathway which proves that CTKs and ET can interact with one other [29]. CIN5 encodes an Activator protein 1 (AP-1) transcription factor with a basic leucine zipper domain that binds to a consensus cis-acting Yap recognition element (YRE) (5'-TTAC/GTAA-3'). Cin5p regulates the osmotic stress response by binding to the promoters of hundreds of genes, including those involved in sugar and alcohol transport and catabolism and synthesis and deposition of glycerol and trehalose. Cin5p is a nuclear protein that is activated by a variety of stressors, including moderate hyperosmotic and oxidative stress, temperature change, and metal exposure. There are many stress-related cis-elements in the CIN5 promoter, including a YRE, four stress response elements, and a heat shock element [30].

\subsection{Signaling Cross Talk}

Genome-profiling experiments with Arabidopsis hormone mutants discovered the presence of a large and dynamic network linking the three major chemical regulators SA, JA, and ET [31]

\subsubsection{JA and $S A$}

During pathogen infection, antagonistic interactions between SAand JA-dependent signaling pathways have been well characterised [32-34]. The SA-mediated defensive reaction generated by infection with the biotrophic fungus Hyaloperonospora arabidopsidis inhibited JA-dependent defences elicited by caterpillar feeding in Arabidopsis [35]. Exogenous administration of SA, on the other hand, suppresses the expression of JA-responsive genes like PDF1.2 and VSP2. The interaction between SA and JA, however, is dosage dependent, since simultaneous treatment with low doses of SA and JA has been found to have synergistic effects on SAand JA-responsive genes [36]. NON EXPRESSOR OF PR-GENES (NPR1), the master regulator of the SA pathway, controls the JA pathway's repression. SA-induced reduction of JA-responsive genes does not need nuclear NPR1 localization, implying that negative JA-signaling effects are facilitated by cytosolic NPR1 [37]. Miao and Zentgraf [38] discovered that JA and SA in Arabidopsis control WRKY53 in Arabidopsis, a senescence-responsive 
transcription factor, in an antagonistic manner, indicating that these two phytohormone pathways intersect during senescence. SA significantly induces WRKY53 expression, and WRKY53 mutations impair SA-mediated responses $[39,40]$. In the nucleus, WRKY53 interacts with the JA-inducible protein epithiospecifying senescence regulator (ESR), which has a detrimental influence on WRKY53's DNA-binding ability. These findings imply that the negative JA/SA crosstalk is mediated during leaf senescence by an interplay between ESR and WRKY53 proteins, which is most likely regulated by the JA/SA equilibrium [38]. By potentiating the SA-dependent expression of PR1 and turning the JA-suppressing actions independent of NPR1, ET modifies the NPR1-dependent JASA antagonism [41]. ET and JA frequently combine in a synergistic manner. The simultaneous activation of JA and ET signaling cascades is required for the production of the JA-responsive gene PDF1.2 [42]. ET also interacts with defences that are dependent on $\mathrm{SA}[43]$.

\subsubsection{JA and ET}

Recent research on JA and ET signaling pathways has shed light on the regulatory mechanisms by which these two senescenceassociated phytohormones control the timing of leaf senescence. Histone deacetylase 6 (HDA6), which deacetylates histone H3, is required for gene silencing and cytosine methylation maintenance showed that HDA6 positively regulates JA-mediated leaf senescence as well as flowering time. Compared with wild-type plants, axe1-5, an Arabidopsis hda6 mutant, and HDA6-NA-interfering (RNAi) plants displayed increased leaf longevity. Expression of the senescence-associated genes SENESCENCE 4 (SEN4) and SAG12 is down-regulated in axe1-5 and HDA6-RNAi plants [44].

The regulatory mechanisms by which these senescence-associated phytohormones govern the time of leaf senescence have been revealed by recent research on the JA and ET signaling pathways. HDA6 is needed for gene silencing and cytosine methylation maintenance because it deacetylates histone H3. HDA6 controls JA-mediated leaf senescence and blooming timing [45,46]. Axe15, an Arabidopsis hda6 mutant, and HDA6-RNAi plants all had longer leaf lifespans than wild-type plants. In axe1-5 and HDA6RNA-interfering (RNAi) plants, expression of the SAGs SEN4 and SAG12 is reduced [44].

ET and JA were found to work together to control the timing of both leaf senescence and floral organ abscission in another study [47]. Ethylene insensitive 2 (EIN2) is a key component of the ET signaling system that serves a variety of functions [47-49]. Exogenous ET had little effect on floral organ abscission in ein21 plants, which is a normal ET response. Exogenous ET had little effect on floral organ abscission in ein2-1 plants, which is a normal ET response. Exogenous MeJA can restore the male-sterile and JA-related phenotype of the dde2-2 mutant with an allene oxide synthase (AOS) mutation [50]. The dde2-2 ein2-1 double-mutants, on the other hand, respond to exogenous ET by abscission of floral organs at a faster pace than wild-type plants. These findings imply that a modest dose of JA can restore ein 2-1 mutants' ET response and show that JA and ET have a unique interaction. [51] revealed that ET signaling is required for JA-induced leaf senescence, particularly the essential ET-signaling components EIN2 and EIN3.
EIN3 temporary activation or overexpression is adequate to speed up the onset of leaf senescence signs. ein3, on the other hand, is a loss-of-function mutant that exhibits prolonged JAinduced leaf senescence. Furthermore, EIN3 binds directly to the promoters of miR164 (microRNA164), repressing EIN3-induced early senescence phenotypesEIN3 functions downstream of EIN2 to inhibit miR164 expression and up-regulate expression of NAC domain containing protein 2 (NAC2), a miR164 target, according to $\mathrm{Li}$ et al. [52]. These findings establish a signaling route involving EIN2-EIN3-miR164-NAC2 in the control of leaf senescence, as well as a mechanistic understanding of the JA and ET signaling pathways' interaction.

Other chemical regulators are known to engage in the defensive cross talk, in addition to the interaction of the primary three defense hormones SA, JA, and ET. SA and JA/ET-dependent defensive responses are known to be attenuated by ABA. ABA suppresses the expression of JA and ET-responsive genes in Arabidopsis [53]. Despite the advancements made in recent years, the bulk of the mechanisms driving hormone cross talk are still unknown.

\section{ROLE OF ESSENTIAL ELEMENTS IN LEAF SENESCENCE}

Plants require key components in order to confirm efficient growth and development in all stages of vegetation and reproduction. Essential's elements commonly present in plants at concentration above $0.1 \%$ of the weight of the dry matter (DMW) $(>1,000 \mathrm{mg}$ $\mathrm{kg}^{-1}$ ) consisting of nitrogen $(\mathrm{N})$, phosphorus $(\mathrm{P})$, potassium $(\mathrm{K})$, sulfur $(\mathrm{S})$, calcium $(\mathrm{Ca})$, and magnesium $(\mathrm{Mg})$ are expressed as macronutrients. Micronutrients like chlorine $(\mathrm{Cl})$, boron, copper, iron $(\mathrm{Fe})$, manganese $(\mathrm{Mn})$, molybdenum $(\mathrm{Mo})$, nickel $(\mathrm{Ni})$, and zinc ( $\mathrm{Zn}$ ) usually have concentrations less than 0.01\% DMW [41]. Essential elements of plant nutrients, along with the atoms $(\mathrm{C})$, hydrogen $(\mathrm{H})$, and oxygen $(\mathrm{O})$, are commonly recognized as indispensable to all plant organisms.

\subsection{Iron}

Iron is very important micronutrient that is required in various processes, viz., DNA synthesis, chlorophyll synthesis and respiration. In plants, iron play's essential role in many biochemical and physiological effects. It is needed for numerous enzymes including the cytochrome of electron transport chain and has a significant part in all of biological processes. Iron is necessary for chlorophyll biosynthesis, chloroplast structure and chloroplast functionality in plants [54]. There is an early rise of leaves senescence in the plants without chlorophyll.

Fe is involved in chlorophyll synthesis and Fe deficiency is associated with chlorosis (yellowing). In chloroplasts and mitochondria, iron containing proteins (heme) cytochromes are found in electron transport systems. Iron is also linked to certain non-heme proteins including ferredoxin [55].

Fe is important to produce redox energy for seedling growth. Free $\mathrm{Fe}$ is, therefore, highly toxic as it creates reactive species of oxygen. Thus, Fe must be closely bound to chelating molecules so that the seed survives without oxidative damage for a long time. Nevertheless, in favorable circumstances, the Fe seed 
stores must easily be removed to convert to active photosynthesis before the seedling can absorb Fe from soil. Seeds constitute an important dietary source of Fe, which is essential for human health. Understanding the Fe-storing mechanisms in seeds is essential for improving Fe supply and reducing Fe deficiency. Crop survival, germination effectiveness and seedling vigor also have a significant effect on Fe content [56].

\subsection{Carbon}

As plant use carbon in gaseous form, high quantity of energy is needed to reduce it before being incorporated into metabolites. Carbon occupies a specific role in the metabolism of plants. Degradation of photosynthetic apparatus during leaf senescence is also an early development leading to a reduction in photosynthetic assimilates production, circulates to sinks and increase the tissue reliance on respiratory metabolism [57].

Elevated $\mathrm{CO}_{2}$ levels and a supplying level of nitrogen change the expression of gene, protein content along with structure, numerous photosynthesis aspects, nitrogen, sugar, and also redox conditions in plants. In plant leaves, increased $\mathrm{CO}_{2}$ usually contributes to sugar accumulation and a decrease in nitrogen content, leading to a $\mathrm{C} / \mathrm{N}$ imbalance in mature leaves, major causes behind premature leaf senescence. Higher $\mathrm{CO}_{2}$ and lower nitrogen activity decrease antioxidant enzymes activity and thus increase development of $\mathrm{H}_{2} \mathrm{O}_{2}$. These modifications contribute to oxidative stress, leading to photosynthetic pigment degradation and ultimately to senescence [58].

\subsection{Nickel}

Nickel, 24th most abundant element, accounts for almost $0.008 \%$ of the earth's crust, so it is natural element of soil and groundwater. $\mathrm{Ni}$ has long been considered a micronutrient, and has a wide range of biological functions [59]. $\mathrm{Ni}$ is a critical micronutrient which helps to turn urea into ammonia and $\mathrm{CO}_{2}$ with the help of the urease enzyme [60]. Most $\mathrm{Ni}$ are translocated and accumulated in leaves during vegetative development. However, most of it is transported to seed during the senescence of leaves, as recorded in soyabean and Mimulus guttatus.

Consequently, any altered Ni content adversely affects plant growth and metabolic processes in plants in various ways. This lack of sufficient micronutrients can affect plant development, plant senescence, and interfere with Fe uptake. Plants with a chlorosis and meristematic necrosis can be observed in the youngest leaves when an insufficient amount of nitrogen is present [36]. Urea toxicity occurs when insufficient nitrogen causes urease activity to become unresponsive, resulting in the buildup of urea in tips of leaves which turns to a necrotic state [61].

Several studies were carried out in regard to assess the importance of $\mathrm{Ni}$ in nitrogen cycle for soybeans. To determine the function of $\mathrm{Ni}$ in plant $\mathrm{N}$-metabolism, urease and the nitrate reductase (NR) activity were assessed. Ni inhibits the enzyme activity, which improves NR production in cucumber, tomato and onion. In addition, the activity of urease has been indicated as a result of $\mathrm{Ni}$ soybean seed therapy and $\mathrm{Ni}$ content in grains, which lead to $\mathrm{N}$ - removal from the old to the young leaves. Reducing nickel soil accessibility may affect N-plant nutrition, organic acid metabolisms, and nitrogen-related enzymes [62].

\subsection{Sulphur}

Sulphur (S) is a macronutrient essential for growth in plants, production, and adaptation to environmental changes. Proteins, coenzymes, vitamins, cysteine, and secondary metabolites such as sulfate flavonoids are needed for biosynthesis [63]. Sulfur is the third nutrient in addition to carbon and nitrogen that is reduced until integration into large biomolecules and macromolecules. It is worth mentioning that plants are able to generate highly oxidized sulfur metabolites. Along with carbon and nitrogen, sulfur is lowmolecular compound. It has been shown that glutathione is the most vital reduced sulfur metabolite that reaches in millimolar concentrations in the chloroplast. At least in some systems, sulfur remobilization in old plant leaves has been observed. It was suggested that both soluble and insoluble sulfur could be recovered from senescing leaves. The research concluded that homoglutathione is the primary export form of processed protein sulfur from senescing soyabean leaves [56].

Sulphide is used as a precursor for methionine synthesis and other key metabolites, including protein storage. Leaves have a double function, first as a primary sink and then as a secondary source in production. Directing crop breeding to decrease glucosinolate levels, as well as storage protein, can result in modified target proteins. At this time, the absence of extensive information explaining the intake, circulation and chance of metabolites containing $\mathrm{S}$ in crops is preventing this approach [64].

\subsection{Potassium}

In addition to nitrogen, the mineral nutrient required by plants is potassium. In cells and tissues, it is extremely mobile via the xylem and phloem cell. In contrast to the mentioned nutrients, potassium is seen not to be metabolized and thus is seen to only form weak complexes where it is simple to exchange. The transport of potassium was the most intensively examined for both physiological and molecular methods in addition to carbohydrates and nitrogen supplies transport [57]. Many plant genes have been identified encoding transporters for $\mathrm{K}^{+}$and most of them have been looks at in depth in heterologous systems like the yeast mutants that are inadequate for transport. The study of $\mathrm{K}^{+}$transport is similarly complex that's because the transports are grouped into (partially) redundant functional multigene family in the situation discussed for the transport of nitrogen.

Potassium has been regularly remobilized from senescent tissues in considerable amounts. However, this factor must be regarded as leaching from tissues easily, in particular senescing tissues. The amount of potassium actually remobilized may therefore be lower than those mentioned in the literature [57].

Potassium is a key nutrient element in photosynthetic metabolism that requires higher amount. The soil potassium deficiency can inhibit photosynthesis and reduce yield in soyabean [65].

When the ABA concentration was raised, $\mathrm{K}$ uptake reduced but starch production rose, according to studies on the effect of $\mathrm{ABA}$ 
in $\mathrm{K}$ uptake and starch formation. Another research supports a link between $\mathrm{K}$, starch, and ABA, showing that increased $\mathrm{K}$ retention in flag leaves lowers ABA levels and inhibits starch breakdown genes, increasing starch stores and enhancing drought stress tolerance [66].

\subsection{Phosphorus}

Phosphorus $(\mathrm{P})$ is an important macronutrient since it is a crucial biomolecule in the construction of key macromolecules including phospholipids and nucleic acids which are involved in the energy metabolism such as pyrophosphate and adenosine triphosphate (ATP). As a result, almost all crucial processes of metabolism in plants, especially photosynthesis and respiration, require P. P is required for nearly all-important metabolic processes in plants, viz, respiration and photosynthesis [67].

Studies showed that remobilization of $\mathrm{P}$ from senescing leaves are scarce. Around half of the pod plus seed $\mathrm{P}$ is provided by leaf $P$ remobilization in typical bean plants. On other hand, it was found by Crafts- Brandner (1992) that no net leaf P remobilization during reproductive growth of soybeans grown in three different $\mathrm{P}$ regimes. As a result, a combination of exogenous and endogenous influences may affect the remobilization of $\mathrm{P}$ as a moving nutrient, rendering it difficult to generalize the importance of its elimination. Nucleic acids form the major storage sites for phosphates, but significant $\mathrm{P}$ quantities also exist in lipids, in both esterified (organic) and (inorganic) phosphate forms, depending on the organisms and growth conditions studied [57]. When nitrogen is "bound" in protein, the action of hydrolytic enzymes affects phosphorus release in nucleic acids. There has been a decline in nucleic acid levels, and an increase in nuclease activities noted in a senescent tissue [68]. Low nutritional phosphorus led to lower leaf $\mathrm{P}$ and increase senescence of leaf. Maintaining a high $\mathrm{P}$ nutrition reduced senescence inducing effect.

\subsection{Nitrogen}

For metabolism of plants, nitrogen is an essential nutrient. Deficiency in nitrogen can cause stress to plants and speed up leaf senescence. In addition to maintaining high photosynthesis levels during filling, high nitrogen levels will delay the senescence of the leaves in non-perennial plants, thus increasing their yield [69]. As a result, leaf senescence should not be regarded as a degenerative process that occurs at the end of the life cycle in non-perennial plants such as soya, wheat, sorghum, and maize, but rather as a metabolic process of deterioration, removal, transportation and also the reserves of cereals which can be increased or delayed through abiotic factors.

During leaf senescence, the primary metabolites of nitrogen and carbohydrates are free amino acids and small peptides. Particularly during seed development, protein and amino acid degradation causes the loss of nutrients, leading to nutrient depletion. During early stages of leaf senescence, the genes responsible for biosynthetic amino acid processes and transport are activated. In comparison, higher sugar levels (glucose, mannose, galactose, and fructose) photosynthesis and respiration rates in wheat plants correspond to late senescence [70]. Although multiple evidence has confirmed that chloroplast proteases are present in senescent leaves were degraded via organelles [68].

Leaf senescence is a natural process that occurs at a later stage of a plant's normal growth and development; however, senescence may be caused in advance when a plant receives insufficient nitrogen from the environment. CTK is a kind of plant hormone that stimulates cell division and differentiation. It is involved in all phases of plant growth and development, including flowering and fruiting. In recent decades, researchers have conducted extensive research on the function of CTK in the prevention of leaf senescence [68-70]. When nitrogen levels are low, plants experience early senescence and poor development, according to research. When nitrate is given to a plant, the CTK content increases in a short and quick manner [71-73], indicating that nitrate deficit may impede the production of CTK and that CTK is implicated in the senescence of plants. To produce CTK, mevalonate and adenosine monophosphate are used as precursors in the synthesis process. When the isopentenyl of dimethylallyl diphosphate is transferred to adenosine, the enzyme isopentenyl transferase (IPT) catalyses the transfer, and the resulting isopentenyl adenosine-5'- triphosphate, isopentenyl adenosine-5'- diphosphate, or isopentenyl adenosine-5'monophosphate [74].

Studies in the overseas have recently revealed that CTK may be produced in aboveground canopy leaves and transferred to the roots via phloem, influencing the root system's nitrogen absorption and assimilation in the soil $[75,76]$. Nitrogen in the soil can boost the efficiency of nitrogen carriers in the root system, enhancing nitrogen absorption, and encouraging CTK synthesis in roots by upregulating the expression of the essential enzyme CTK synthesis IPT gene. CTK are carried from the xylem to the upper canopy by transpiration flow and effectively reach cells to control nitrogen distribution in the canopy, thereby alleviating leaf senescence and increasing canopy leaf photosynthetic capability [77].

\subsection{Cobalt}

Cobalt $(\mathrm{Co})$ is a trace element that is not abundant in nature; its concentration in soils ranges between 15 and 25 parts per million (ppm), and it is around $0.04 \mathrm{ppm}$ in natural water [71]. In higher plants, Co has been shown to strongly bind to roots and to be primarily absorbed from soil solutions through passive transport [78]. Because of their similarity to nickel (Ni), it is possible that the two elements enter cells through the same plasma membrane carriers $[79,80]$. Other beneficial effects of Co have been reported, including the prevention of leaf senescence through inhibition of ET biosynthesis and the improvement of drought resistance in seeds. Co also increased the accumulation of isoquinoline (an alkaloid) in medicinal plants by upregulating the production of aromatic amino acid precursors of alkaloids [81], which was previously shown to be beneficial. This final impact may imply that Co might indirectly increase biotic stress tolerance, however this theory has not been tested to this point. As has been shown for other hyperaccumulators of elements, the high tissue Co levels in hyperaccumulators of Co may also provide direct protection against herbivory or diseases in these plants. 


\subsection{Magnesium, Calcium, Selenium and Micronutrients}

Magnesium $(\mathrm{Mg})$ is a macro-element that is required for life and is often found in cells as a divalent cation. $\mathrm{Mg}^{2+}$ is a cofactor of enzyme function in conjunction with ATP, a stabilizer for the structure of the ribosomal RNA polymerase, and the central atom in chlorophyll [82-84]. Mg homeostasis in the plant should be tightly managed in order for metabolic activities to run smoothly. According to an ionomic investigation of Arabidopsis M3 mutants, Mg concentrations had the lowest variation across leaves of ambient samples [82] and the lowest variance among 18 minerals in Arabidopsis M3 mutants [85]. In the environment, magnesium is plentiful, with more than 20,000 ppm in the earth's crust, making it the eighth most abundant element on the planet. Despite the abundance of magnesium in the soil, it is frequently deficient in the field due to soil acidity and competition with other cations such as hydrogen $\left(\mathrm{H}^{+}\right)$, potassium $\left(\mathrm{K}^{+}\right)$, calcium $\left(\mathrm{Ca}^{2+}\right)$, and ammonium $\left(\mathrm{NH}^{4+}\right)$ in the soil [86-90], particularly in very sandy soils and in the cultivation of tobacco and potatoes [85-86]. There have been various studies that have identified the signs and symptoms of magnesium shortage in crops [61-65]. Under typical circumstances, the magnesium concentration of the vegetative portions is between $0.15 \%$ and $0.35 \%$ of the dry weight of the plant [77]. When the magnesium level falls below this threshold, symptoms appear on the leaves. Symptoms are more likely to manifest themselves during the time of grain filling or fruit growth, which has an impact on the quality and quantity of the agricultural output $[90,91]$. Foliar tissues between the green veins of magnesium-deficient leaves are yellowish to bronze in color, orange-yellow in color, or reddish in color [86-90], and they may also show brown interveinal necrosis. When $\mathrm{Mg}$ availability is restricted, adult leaves senesce at a quicker rate than juvenile leaves in general.

The collection of carbohydrates in source leaves caused by $\mathrm{Mg}$ deficiency results in changes in photosynthetic carbon metabolism and a reduction in $\mathrm{CO}_{2}$ fixation, which is likely to result in the production of ROS via spillage of electrons and absorbed energy from photosynthesis. Plant hormone levels and signaling are affected by a magnesium deficit. Mg shortage has been shown to enhance ET, a plant hormone involved in fruit ripening, leaf senescence, and stress response signaling [92].

Plants respond to $\mathrm{Mg}$ shortage in a variety of ways, the majority of which are linked to coping with the difficulties that arise when $\mathrm{Mg}$ is absent, such as chlorophyll degradation and antioxidation. Plant hormone levels and signaling are affected by magnesium shortage. $\mathrm{Mg}$ shortage has been shown to enhance ET, a plant hormone involved in fruit ripening, leaf senescence, and stress response signaling. In Arabidopsis, the genes encoding ET biosynthetic enzymes were also increased, as were the genes encoding ET biosynthetic enzymes in rice [93]. Interestingly, moderate drying enhanced ET production in tomato leaves [94,95]. Furthermore, ABA signaling might be one of Mg deficiency's major targets. Half of the elevated genes in leaves with short-term $\mathrm{Mg}$ shortage were similarly ABA-responsive in Arabidopsis, according to Sobeih et al. [96]. Short-term Mg shortage impacted the central oscillator of the circadian clock in roots, which might be implicated in a component of ABA and ET signaling that is reliant on the circadian clock or circadian rhythm [97].

Calcium has been found to increase in senescing leaves over and over again, which is the least moving of all macronutrients. In view of the possibility that senescence may be a consequence of the deterioration of cell walls and membrane compartments, calcium was studied by Poovaiah [7] as a possible agent which might defer senescence. Calcium treatment delayed leaf senescence in corn leaf discs. The effect of calcium on senescence was additive to the CTK deferral of senescence. An increase in hydraulic permeability to water during senescence was demonstrated and this increase was deferred by calcium.

Although selenium (Se) is a rare element in soil, there is increasing evidence that it can protect plants against biotic stressors. Se's ability to defend from biotic stressors might be due to a mix of direct and indirect effects. Se treatment has also been found to increase JA and ET synthesis in plants, as well as the development of defense-related proteins and sulfate/selenate, as a result, low-dose Se fertilization may stimulate plant growth while also increasing tolerance to pests and other stressors [98].

Information on micronutrient removal does not provide a widespread image. Several have been documented for remobilization and accumulation in senescent leaves including $\mathrm{Fe}$, $\mathrm{Cu}, \mathrm{Mn}$, and $\mathrm{Zn}[57]$.

\section{CURRENT APPLICATIONS}

In last few years, substantial progress was achieved using molecular biology techniques to investigate events which occur during leaf senescence. Many of the genes that have been identified for the senescence process, and some of encoded protein's roles have proven to be entirely unexpected. Transgenic plant research has begun to provide a new outlook on the processes that take place during senescence to determine the functions of others. A clearer picture of the regulatory mechanisms involved in senescence was obtained through the availability of genes, promoters and variations [99].

Senescence of leaf is very critical for agricultural plants because it reduces yield, biomass, and alters nutrient value. Due to its general prevalence, interest in plant breeding programs have arisen a desire to better understand its molecular mechanisms, particularly regulation. The technique of CTK promoter's synthesis in senescent leaves by over expressing IPT under the guidance of senescencecontrolled promoters is the most prominent biotechnology application of leaf senescence for crop productivity [99]. The use of key transcription factors like NAC-like transcription factor, activated by AP3/P1." (NAP) and Eukaryotic translation initiation factor 5A-1 (elF-5A) (translation initiation factor) to manipulate senescence has been shown to be very successful and has a lot of commercial potential [100].

\section{CONCLUSION}

Leaf senescence is considered as a dynamic process where different environmental signals are incorporated into age based developmental pathways. Therefore, mechanisms are needed to 
determine both the age of cells, organs as well as environmental factors, incorporate these factors, select pathway and ultimately execute them. Molecular research has been carried out on the basis of these crucial steps and will remain a significant field of study in the future. However, it is more difficult to report how plants temporarily and spatially organize these processes during senescence. The establishment of a phenomenon system and the integrated data with genomic, proteomic, and metabolomic data will be a new technological challenge to resolve this problem, linking genetical and all environmental input to be included in plant and seed production during the whole growth cycle.

\section{FUTURE PROSPECTIVES}

Cellular senescence is a time-dependent transition rather than a single discrete period of cellular physiology and metabolism. As a result, senescence must be known even from a complex time perception. The phase of senescence is anticipated also to involve cell to cell and organ to organ contact, which is spatially organized. Therefore, a paradigm shift in the analysis and description of the process might be necessary to fully understand leaf senescence.

It should also be stated that leaf cell decomposes and die as they mature. However, individual cells in a senescing leaf are extremely diverse. It raises the question of how this highly diversity in leaf decay occurs along with leaf senescence. Physiological and developmental states of the entire plant can have an effect on the rate of leaf senescence. It is also very crucial to consider the systemic integration of senescence and death within the whole plant. Seed setting and leaf senescence regulatory elements are yet unknown to us in terms of how they are combined with plant productivity. Another important aspect which has not been discovered is the relocation of nutrients from senescent leaves.

In addition, leaf senescence is a developmental technique that has developed in an evolutionary way, and we can therefore expect to see different physiology and regulatory nodes for plants which have evolved in different environment. Comparing observations of various Arabidopsis ecotypes and senescence plants using a particular approach will be extremely useful in this regard. This allows one to investigate metabolism and physiological mechanisms, as well as their regulators, senescence and death process to be described as networks of the molecular, cellular, and organs. The terms molecular and system levels are essential to comprehend the different processes in biology including aging, senescence and death.

\section{ACKNOWLEDGEMENTS}

All authors made substantial contributions to conception and design, acquisition of data, and took part in drafting of this review article.

\section{CONFLICT OF INTEREST}

The authors declares that there is no conflict of interest.

\section{FUNDING}

There is no funding to this research.

\section{ETHICAL APPROVALS}

This study does not involve experiments on animals or human subjects.

\section{DATA AVAILABILITY}

All data generated and analyzed are included within this research article.

\section{PUBLISHER'S NOTE}

This journal remains neutral with regard to jurisdictional claims in published

institutional affiliation.

\section{REFERENCES}

1. Woo HR, Kim HJ, Lim PO, Nam HG. Leaf senescence: systems and dynamics aspects. Annu Rev Plant Biol 2019;70(1):347-76; doi:10.1146/annurev-arplant-050718-095859.

2. Shahri W, Tahir I. Flower senescence: some molecular aspects. Planta 2014;239(2):277-97; doi:10.1007/s00425-013-1984-z.

3. Lim PO, Woo HR, Nam HG. Molecular genetics of leaf senescence in Arabidopsis. Trends Plant Sci 2003;8(6):272-8; doi:10.1016/S13601385(03)00103-1.

4. Tripathi SK, Tuteja N. Integrated signaling in flower senescence: an overview. Plant Signal Behav 2007;2(6):437-45; doi:10.4161/ psb.2.6.4991.

5. Mayta ML, Hajirezaei MR, Carrillo N, Lodeyro AF. Leaf senescence: the chloroplast connection comes of age. Plants (Basel) 2019;8(11):495; doi:10.3390/plants8110495

6. Pennazio S. Plant senescence. A complex lesson of biology of development. Riv Biol 2009;102(3):399-419.

7. Poovaiah BW. Role of calcium in ripening and senescence. Commun Soil Sci Plant Anal 1979;10(1):83-8.

8. Woo HR, Kim HJ, Nam HG, Lim PO. Plant leaf senescence and death - regulation by multiple layers of control and implications for aging in general. J Cell Sci 2013;126(21):4823-33; doi:10.1242/ jes.109116.

9. Woo HR, Masclaux-Daubresse C, Lim PO. Plant senescence: how plants know when and how to die. J Exp Bot 2018;69(4):715-8; doi:10.1093/jxb/ery011.

10. Bresson J, Bieker S, Riester L, Doll J, Zentgraf U. A guideline for leaf senescence analyses: from quantification to physiological and molecular investigations. J Exp Bot 2018;69(4):769-86; doi:10.1093/ $\mathrm{jxb} / \mathrm{erx} 246$.

11. $\mathrm{Lu} \mathrm{H}$, Gordon MI, Amarasinghe V, Strauss SH. Extensive transcriptome changes during seasonal leaf senescence in fieldgrown black cottonwood (Populus trichocarpaNisqually-1). Sci Rep 2020;10(1):6581; doi:10.1038/s41598-020-63372-2.

12. Yoshida S. Molecular regulation of leaf senescence. Curr Opin Plant Biol 2003;6(1):79-84; doi:10.1016/S1369526602000092.

13. Munné-Bosch S, Alegre L. Die and let live: leaf senescence contributes to plant survival under drought stress. Funct Plant Biol 2004;31(3):203-16; doi:10.1071/FP03236.

14. Wasternack C, Hause B. Jasmonates: biosynthesis, perception, signal transduction and action in plant stress response, growth and development. An update to the 2007 review in Annals of Botany. Ann Bot 2013;111(6):1021-58; doi:10.1093/aob/mct067. 
15. Buchanan-Wollaston V, Earl S, Harrison E, Mathas, E, Navabpour S, Page T, et al. The molecular analysis of leaf senescence- a genomics approach. Plant Biotechnol J 2003;1(1):3-22; doi:10.1046/j.14677652.2003.00004.x.

16. Hunter DA, Yoo SD, Butcher SM, McManus MT. Expression of 1-aminocyclopropane-1-carboxylate oxidase during leaf ontogeny in white clover. Plant Physiol 1999;120(1):131-42; doi:10.1104/ pp.120.1.131, Pubmed:10318691.

17. Kende H. Ethylene biosynthesis. Annu Rev Plant Physiol Plant Mol Biol 1993;44(1):283-307; doi:10.1146/annurev.pp.44.060193.001435.

18. Johnson PR, Ecker JR. The ethylene gas signal transduction pathway: a molecular perspective. Annu Rev Genet 1998;32:227-54; doi:10.1146/annurev.genet.32.1.227.

19. Surplus SL, Jordan BR, Murphy AM, Carr JP, Thomas B, Mackerness SAH. Ultraviolet-B-induced responses in Arabidopsis thaliana: role of salicylic acid and reactive oxygen species in the regulation of transcripts encoding photosynthetic and acidic pathogenesis-related proteins. Plant Cell Environ 1998;21(7):685-94; doi:10.1046/j.13653040.1998.00325.x.

20. Orozco-Cardenas M, Ryan CA. Hydrogen peroxide is generated systemically in plant leaves by wounding and systemin via the octadecanoid pathway. Proc Natl Acad Sci U S A 1999;96(11):6553-7; doi:10.1073/pnas.96.11.6553.

21. Pellinen R, Palva T, Kangasjärvi J. Short communication: subcellular localization of ozone-induced hydrogen peroxide production in birch (Betula pendula) leaf cells. Plant J 1999;20(3):349-56; doi:10.1046/ j.1365-313x.1999.00613.x.

22. Häffner E, Konietzki S, Diederichsen E. Keeping control: the role of senescence and development in plant pathogenesis and defense. Plants (Basel) 2015;4(3):449-88; doi:10.3390/plants4030449.

23. Gazzarrini S, McCourt P. Genetic interactions between ABA, ethylene and sugar signaling pathways. Curr Opin Plant Biol 2001;4(5):38791; doi:10.1016/s1369-5266(00)00190-4.

24. Pieterse CMJ, Van der Does D, Zamioudis C, Leon-Reyes A, Van Wees SCM. Hormonal modulation of plant immunity. Annu Rev Cell Dev Biol 2012;28:489-521; doi:10.1146/annurev-cellbio-092910-154055.

25. Vanhee $\mathrm{C}$, Batoko H. Autophagy involvement in responses to abscisic acid by plant cells. Autophagy 2011;7(6):655-6; doi:10.4161/ auto.7.6.15307.

26. Fischer AM. The complex regulation of senescence. Crit Rev Plant Sci 2012;31(2):124-47; doi:10.1080/07352689.2011.616065.

27. Zhao Y. Auxin biosynthesis and its role in plant development. Annu Rev Plant Biol 2010;61:49-64; doi:10.1146/annurevarplant-042809-11230.

28. Kim J, Kim JH, Lyu JI, Woo HR, Lim PO. New insights into the regulation of leaf senescence in Arabidopsis. J Exp Bot 2018;69(4):787-99; doi:10.1093/jxb/erx287.

29. Pereira J, Pimentel C, Amaral C, Menezes RA, Rodrigues-Pousada C. Yap4 PKA- and GSK3-dependent phosphorylation affects its stability but not its nuclear localization. Yeast (Chichester Engl) 2009;26(12):641-53; doi:10.1002/yea.1711

30. Hanlon SE, Rizzo JM, Tatomer DC, Lieb JD, Buck MJ. The stress response factors Yap6, Cin5, Phd1, and Skn7 direct targeting of the conserved co-repressor Tup1-Ssn6 in S. cerevisiae. PLoS One 2011;6(4):e19060; doi:10.1371/journal.pone.0019060

31. Glazebrook J, Chen W, Estes B, Chang HS, Nawrath C, Métraux JP, et al. Topology of the network integrating salicylate and jasmonate signal transduction derived from global expression phenotyping. Plant J 2003;34(2):217-28.

32. Cui J, Bahrami AK, Pringle EG, Hernandez-Guzman G, Bender CL, et al. Ausubel Pseudomonas syringae manipulates systemic plant defenses against pathogens and herbivores. Proc Natl Acad Sci U S A 2005;102(5):1791-6; doi:10.1073/pnas.0409450102

33. Glazebrook J. Contrasting mechanisms of defense against biotrophic and necrotrophic pathogens. Annu Rev Phytopathol 2005;43:205-27; doi:10.1146/annurev.phyto.43.040204.135923.
34. Spoel SH, Dong X. Making sense of hormone crosstalk during plant immune responses. Cell Host Microbe 2008;3(6):348-51.

35. Koornneef A, Leon-Reyes A, Ritsema T, Verhage A, Den Otter FC, van Loon LC, et al. Kinetics of salicylate-mediated suppression of jasmonate signaling reveal a role for redox modulation. Plant Physiol 2008;147(3):1358-68.

36. Schenk PM, Kazan K, Wilson I, Anderson JP, Richmond T, Somerville SC, et al. Coordinated plant defense responses in Arabidopsis revealed by microarray analysis. Proc Natl Acad Sci U S A 2000;97(21):11655-60.

37. Spoel SH, Koornneef A, Claessens SM, Korzelius JP, Van Pelt JA, Mueller MJ, et al. NPR1 modulates cross-talk between salicylate- and jasmonate-dependent defense pathways through a novel function in the cytosol. Plant Cell 2003;15(3):760-70.

38. Miao Y, Zentgraf U. The antagonist function of Arabidopsis WRKY53 and ESR/ESP in leaf senescence is modulated by the jasmonic and salicylic acid equilibrium. Plant Cell 2007;19(3):819-30; doi:10.1105/ tpc. 106.042705

39. Miao Y, Laun T, Zimmermann P, Zentgraf U. Targets of the WRKY53 transcription factor and its role during leaf senescence in Arabidopsis. Plant Mol Biol 2004;55(6):853-67; doi:10.1007/s11103-004-2142-6

40. Hu Y, Dong Q, Yu D. Arabidopsis WRKY46 coordinates with WRKY70 and WRKY53 in basal resistance against pathogen Pseudomonas syringae. Plant Sci 2012;185-6:288-97; doi:10.1016/j. plantsci.2011.12.003

41. Leon-Reyes A, Spoel SH, De Lange ES, Abe H, Kobayashi M, Tsuda $\mathrm{S}$, et al. Ethylene modulates the role of NONEXPRESSOR OF PATHOGENSIS-RELATED GENES1 in cross talk between salicylate and jasmonate signaling. Plant Physiol 2009;149(4):1797-809.

42. Penninckx IA, Thomma BP, Buchala A, Métraux JP, Broekaert WF. Concomitant activation of jasmonate and ethylene response pathways is required for induction of a plant defensin gene in Arabidopsis. Plant Cell 1998;10(12):2103-13.

43. Verberne MC, Hoekstra J, Bol JF, Linthorst HJ. Signaling of systemic acquired resistance in tobacco depends on ethylene perception. Plant $\mathrm{J}$ 2003;35(1):27-32.

44. Wu K, Zhang $\mathrm{L}$, Zhou $\mathrm{C}$, Yu CW, Chaikam V. HDA6 is required for jasmonate response, senescence and flowering in Arabidopsis. J Exp Bot 2008;59(2):225-34; doi:10.1093/jxb/erm300

45. Lippman Z, May B, Yordan C, Singer T, Martienssen R. Distinct mechanisms determine transposon inheritance and methylation via small interfering RNA and histone modification. PLOS Biol 2003;1(3):E67; doi:10.1371/journal.pbio.0000067

46. Earley K, Lawrence RJ, Pontes O, Reuther R, Enciso AJ, Silva M, et al. Erasure of histone acetylation by Arabidopsis HDA6 mediates large-scale gene silencing in nucleolar dominance. Genes Dev 2006;20(10):1283-93; doi:10.1101/gad.1417706

47. Kim J, Patterson SE, Binder BM. Reducing jasmonic acid levels causes ein2 mutants to become ethylene responsive. FEBS Lett 2013;587(2):226-30; doi:10.1016/j.febslet.2012.11.030

48. Alonso JM, Hirayama T, Roman G, Nourizadeh S, Ecker JR. EIN2, a bifunctional transducer of ethylene and stress responses in Arabidopsis. Science 1999;284(5423):2148-52; doi:10.1126/ science. 284.5423 .2148

49. Binder BM, Mortimore LA, Stepanova AN, Ecker JR, Bleecker AB. Short-term growth responses to ethylene in Arabidopsis seedlings are EIN3/EIL1 independent. Plant Physiol 2004;136(2):2921-7; doi:10.1104/pp.104.050393

50. Dong J, Chen C, Chen Z. Expression profiles of the Arabidopsis WRKY gene superfamily during plant defense response. Plant Mol Biol 2003;51(1):21-37; doi:10.1023/a:1020780022549

51. Li C, Liu G, Xu C, Lee GI, Bauer P, Ling HQ, et al. The tomato suppressor of prosystemin-mediated responses 2 gene encodes a fatty acid desaturase required for the biosynthesis of jasmonic acid and the production of a systemic wound signal for defense gene expression. Plant Cell 2003;15(7):1646-61; doi:10.1105/tpc.012237 
52. Li Z, Peng J, Wen X, Guo H. Ethylene-insensitive3 is a senescenceassociated gene that accelerates age-dependent leaf senescence by directly repressing miR164 transcription in Arabidopsis. Plant Cell 2013;25(9):3311-28; doi:10.1105/tpc.113.113340

53. Anderson JP, Badruzsaufari E, Schenk PM, Manners JM, Desmond OJ, Ehlert $\mathrm{C}$, et al. Antagonistic interaction between abscisic acid and jasmonate-ethylene signaling pathways modulates defense gene expression and disease resistance in Arabidopsis. Plant Cell 2004;16(12):3460-79.

54. Rout GR, Sahoo S. Role of iron in plant growth and metabolism. Rev Agric Sci 2015;3:1-24; doi:10.7831/ras.3.1.

55. Hochmuth G. Iron (Fe) nutrition of plants. Soil Water Sci 2011;2011(8). EDIS. Available via https://edis.ifas.ufl.edu/ss555. 2018.

56. Mari S, Bailly C, Thomine S. Handing off iron to the next generation: how does it get into seeds and what for? Biochem J 2020;477(1):259 74; doi:10.1042/BCJ20190188.

57. Fischer, AM. Nutrient remobilization during leaf senescence. In: Gan S (ed.). Annual Plant Reviews, Senescence Processes in Plants, Blackwell Publishing Ltd, Oxford, UK, vol. 26, pp 87-107, 2007; doi:10.1002/9780470988855.ch5.

58. Agüera E, De la Haba P. Leaf senescence in response to elevated atmospheric $\mathrm{CO}_{2}$ concentration and low nitrogen supply. Biol Plant 2018;62(3):401-8; doi:10.1007/s10535-018-0798-z.

59. Ahmad MSA, Ashraf M. Essential roles and hazardous effects of nickel in plants. In: Whitacre DM (ed.). Reviews of Environmental Contamination and Toxicology, Springer, New York, NY, vol. 214, pp 125-67, 2012; doi:10.1007/978-1-4614-0668-6_6.

60. Amjad M, Raza H, Murtaza B, Abbas G, Imran M, Shahid M, et al. Nickel toxicity induced changes in nutrient dynamics and antioxidant profiling in two maize (Zea mays L.) hybrids. Plants (Basel) 2019;9(1):5; doi:10.3390/plants9010005.

61. Fabiano CC, Tezotto T, Favarin JL, Polacco JC, Mazzafera P. Essentiality of nickel in plants: a role in plant stresses. Front Plant Sci 2015;6:754; doi:10.3389/fpls.2015.00754.

62. de Macedo FG, Bresolin JD, Santos EF, Furlan F, Lopes da Silva WT, Polacco JC, et al. Nickel availability in soil as influenced by liming and its role in soybean nitrogen metabolism. Front Plant Sci 2016;7:1358; doi:10.3389/fpls.2016.01358.

63. Gigolashvili T, Kopriva S. Transporters in plant sulfur metabolism. Front Plant Sci 2014;5:442; doi:10.3389/fpls.2014.00442.

64. Borpatragohain P, Rose TJ, Liu L, Barkla BJ, Raymond CA, King GJ. Remobilization and fate of sulphur in mustard. Ann Bot 2019;124(3):471-80; doi:10.1093/aob/mcz101.

65. Wang $\mathrm{X}$, Zhao $\mathrm{X}$, Jiang $\mathrm{C}, \mathrm{Li} \mathrm{C}$, Cong $\mathrm{S}$, WuD, et al. Effects of potassium deficiency on photosynthesis and photoprotection mechanisms in soybean (Glycine max (L.) Merr.). J Integr Agric 2015;14(5):856-63; doi:10.1016/S2095-3119(14)60848-0.

66. Hosseini SA, Hajirezaei MR, Seiler C, Sreenivasulu N, von Wirén $\mathrm{N}$. A potential role of flag leaf potassium in conferring tolerance to drought-induced leaf senescence in barley. Front Plant Sci 2016;7:206.

67. Stigter KA, Plaxton WC. Molecular mechanisms of phosphorus metabolism and transport during leaf senescence. Plants (Basel) 2015;4(4):773-98; doi:10.3390/plants4040773.

68. Wen B, Xiao W, Mu Q, Dongmei Li, Xiude C, Hongyu W, et al. How does nitrate regulate plant senescence? Plant Physiol Biochem 2020;157:60-9; doi:10.1016/j.plaphy.2020.08.041.

69. Bushnell WR. Delay of senescence in wheat leaves by cytokinins, nickel, and other substances. Can J Bot 1966;44(11):1485-93.

70. JanečkováH, HusičkováA, FerrettiU, Prčina M, Pilařová E, Plačková $\mathrm{L}$, et al. The interplay between cytokinins and light during senescence in detached Arabidopsis leaves. Plant Cell Environ 2018;41(8):1870 85; doi:10.1111/pce.13329.

71. Osugi A, Kojima M, Takebayashi Y, Ueda N, Kiba T, Sakakibara H. Systemic transport of trans-zeatin and its precursor have differing roles in Arabidopsis shoots. Nat Plants 2017;24(3):17112; doi: 10.1038/ nplants.2017.112.
72. Takei K, Ueda N, Aoki K, Kuromori T, Hirayama T, Shinozaki K, et al. AtIPT3 is a key determinant of nitrate-dependent cytokinin biosynthesis in Arabidopsis. Plant Cell Physiol 2004;45(8):1053-62; doi: $10.1093 / \mathrm{pcp} / \mathrm{pch} 119$.

73. Sakakibara H, Takei K, Hirose N. Interactions between nitrogen and cytokinin in the regulation of metabolism and development. Trends Plant Sci 2006;11(9):440-8; doi:10.1016/j.tplants.2006.07.004.

74. Wang R, Tischner R, Gutiérrez RA, Hoffman M, Xing X, Chen M, et $a l$. Genomic analysis of the nitrate response using a nitrate reductasenull mutant of Arabidopsis. Plant Physiol 2004;136(1):2512-22; doi:10.1104/pp.104.044610.

75. Kudo T, Kiba T, Sakakibara H. Metabolism and long-distance translocation of cytokinins. J Integr Plant Biol 2010;52(1):53-60.

76. Lacombe B, Achard P. Long-distance transport of phytohormones through the plant vascular system. Curr Opin Plant Biol 2016;34:1-8.

77. Zürcher E, Müller B. Cytokinin synthesis, signaling, and functionadvances and new insights. Int Rev Cell Mol Biol 2016;324:1-38.

78. Buchanan BB. Role of light in the regulation of chloroplast enzymes Annu Rev Plant Physiol 1980;31(1):341-74.

79. Marschner H. Functions of mineral nutrients: micronutrients. In: Mineral Nutrition in Higher Plants, 2nd edition, Academic Press, London, UK, 1995, pp 313-404.

80. Wyttenbach A, Furrer V, Tobler L. The concentration ratios plant to soil for the stable elements Cs, Rb and K. Sci Total Environ 1995; 173 4:361-7; doi:10.1016/0048-9697(95)04737-9.

81. Lahner B, Gong J, Mahmoudian M, Smith EL, Abid KB, Rogers $\mathrm{EE}$, et al. Genomic scale profiling of nutrient and trace elements in Arabidopsis thaliana. Nat Biotechnol 2003;21(10):1215-21; doi:10.1038/nbt865.

82. Kobayashi NI, Iwata N, Saito T, Suzuki H, Iwata R, Tanoi K, et al. Application of ${ }^{28} \mathrm{Mg}$ for characterization of $\mathrm{Mg}$ uptake in rice seedling under different $\mathrm{pH}$ conditions. J Radioanal Nucl Chem 2013;296(1):531-34; doi:10.1007/s10967-012-2010-9.

83. Jacoby B. Calcium-magnesium ratios in the root medium as related to magnesium uptake by citrus seedlings. Plant Soil 1961;15(1):74-80; doi:10.1007/BF01421750.

84. Tanaka H, Ougimoto T, Sahashi H. Effects of calcium and magnesium of nutrient solution on their composition in leaves of tomato seedlings. Jpn J Soil Sci Plant Nutr 1991;62:507-11.

85. Bennett WF. Better crops. In: Bennett WF (ed.). Nutrient Deficiencies and Toxicities in Crop, APS Publishing, St Paul, MN, vol. 95, 2011, pp 5-6.

86. Uchida R. Essential nutrients for plant growth: nutrient functions and deficiency symptoms. In: Silva JA, Uchida R (eds.). College of Tropical Agriculture and Human Resources, University of Hawaii, Honolulu, HI, 2000, pp 31-55.

87. Tamburini G, Berti A, Morari F, Marini L. Degradation of soil fertility can cancel pollination benefits in sunflower. Oecologia 2016;180(2):581-7; doi:10.1007/s00442-015-3493-1.

88. Aitken RL, Dickson T, Hailes KJ, Moody PW. Response of fieldgrown maize to applied magnesium in acidic soils in north-eastern Australia. Aust J Agric Res 1999;50(2):191-8; doi:10.1071/A98149.

89. Moss GI, Higgins ML. Magnesium influences on the fruit quality of sweet orange (Citrus sinensis L. osbeck). Plant Soil 1974;41(1):10312; doi:10.1007/BF00017948.

90. Hariadi Y, Shabala S. Screening broad beans (Vicia faba) for magnesium deficiency. I. Growth characteristics, visual deficiency symptoms and plant nutritional status. Funct Plant Biol 2004;31(5):529-37; doi:10.1071/FP03201.

91. Palit S, Sharma A, Talukder G. Effect of cobalt on plants. Bot Rev 1994;60(2):149-81.

92. Baxter IR, Vitek O, Lahner B, Muthukumar B, Borghi M, Morrissey $\mathrm{J}$, et al. The leaf ionome as a multivariable system to detect a plant's physiological status. Proc Natl Acad Sci U S A 2008;105:12081-6; doi:10.1104/pp.15.01971 
93. 93. Hermans C, Vuylsteke M, Coppens F,Cristescu SM, Harren F, Verbruggen N. Systems analysis of the responses to long-term magnesium deficiency and restoration in Arabidopsis thaliana. $\mathrm{N}$ Phytol 2010;187(1):132-44; doi:10.1111/j.1469-8137.2010.03257.x.

94. Verbruggen N, Hermans C. Physiological and molecular responses to magnesium nutritional imbalance in plants. Plant Soil 2013;368(1-2):87-99.

95. Wilkinson S, Davies WJ. Drought, ozone, ABA and ethylene: new insights from cell to plant to community. Plant Cell Environ 2010;33(4):510-25.

96. Hermans C, Vuylsteke M, Coppens F, Craciun A, Inzé D, Verbruggen N. Early transcriptomic changes induced by magnesium deficiency in Arabidopsis thaliana reveal the alteration of circadian clock gene expression in roots and the triggering of abscisic acid-responsive genes. New Phytol 2010;187(1):119-31.

97. Sobeih WY, Dodd IC, Bacon MA, Grierson D, Davies WJ. Longdistance signals regulating stomatal conductance and leaf growth in tomato (Lycopersicon esculentum) plants subjected to partial rootzone drying. J Exp Bot 2004;55(407):2353-63.
98. Pilon-Smits EA, Quinn CF, Tapken W, Malagoli M, Schiavon M. Physiological functions of beneficial elements. Curr Opin Plant Biol 2009;12(3):267-74; doi:10.1016/j.pbi.2009.04.009

99. Tanoi K, Kobayashi NI. Leaf senescence by magnesium deficiency. Plants 2015;4(4):756-72; doi:10.3390/plants4040756

100. Guo Y, Gan SS. Translational researches on leaf senescence for enhancing plant productivity and quality. J Exp Bot 2014;65(14):390113; doi:10.1093/jxb/eru248.

\section{How to cite this article:}

Singh S, Singh M, Bisht S, Sharma JG. Leaf senescence and its regulation with phytohormones and essential elements: An overview. J Appl Biol Biotech 2022; 10(02): $185-197$. 